\title{
On an invariance principle for phase separation lines
}

\author{
Lev Greenberg, Dmitry Ioffe* \\ Faculty of Industrial Engineering, Technion, Haifa 3200, Israel
}

Received 22 April 2004; accepted 22 July 2004

Available online 29 June 2005

\begin{abstract}
We prove invariance principles for phase separation lines in the two dimensional nearest neighbour Ising model up to the critical temperature and for connectivity lines in the general context of high temperature finite range ferromagnetic Ising models. ○ 2005 Elsevier SAS. All rights reserved.
\end{abstract}

\section{Résumé}

Nous démontrons des principes d'invariance d'une part pour les lignes de séparation de phase, dans le cas du modèle d'Ising bidimensionnel à plus proches voisins jusqu'à la température critique, et d'autre part pour les lignes de connectivité dans le contexte général des modèles d'Ising ferromagnétiques à portée finie, pour les hautes températures.

(c) 2005 Elsevier SAS. All rights reserved.

MSC: 60F15; 60K $15 ; 60 \mathrm{~K} 35 ; 82 \mathrm{~B} 20 ; 37 \mathrm{C} 30$

Keywords: Ising model; Random path representation; Ornstein-Zernike decay of correlations; Brownian bridge; Ruelle operator;

Renormalization; Local limit theorems

\section{Introduction and results}

\subsection{Phase separation lines}

A canonical example of phase separation lines is given in the framework of the nearest neighbour two dimensional Ising model below the critical temperature $T_{c}$. It would be convenient to draw these lines through the bonds

\footnotetext{
* Partly supported by the EU Network Postdoctoral Training Program in Mathematical Analysis of Large Quantum Systems under the contract HPRN-CT-2002-00277 and by the Senior Visiting Fellowship at the Isaac Newton Institute in the framework of the IGS Programme.

* Corresponding author.

E-mail address: ieioffe@ie.technion.ac.il (D. Ioffe).
} 
of the integer lattice $\mathbb{Z}^{2}$, which means that the corresponding spins should live on the vertices of the shifted dual lattice $\mathbb{Z}^{2 *}=(1 / 2,1 / 2)+\mathbb{Z}^{2}$. Let

$$
\mathbf{S}_{M}^{*}=(1 / 2,1 / 2)+[0, \ldots, M-1] \times \mathbb{Z} \subset \mathbb{Z}^{2 *}
$$

be an infinite dual strip of width $M$. A spin configuration $\sigma$ on $\mathbf{S}_{M}^{*}$ is an element of $\{-1,1\}^{\mathbf{S}_{M}^{*}}$. Given a unit vector $\mathfrak{n} \in \mathbb{S}^{1}$ with $\mathfrak{n}_{1} \geqslant 1 / \sqrt{2}$ consider the so called Dobrushin's boundary conditions

$$
\bar{\sigma}^{\mathfrak{n}}(y)= \begin{cases}+1, & \text { if }\left(\mathfrak{n}^{\perp}, y\right)_{2} \geqslant 0 \\ -1, & \text { if }\left(\mathfrak{n}^{\perp}, y\right)_{2}<0\end{cases}
$$

where $\mathfrak{n}^{\perp}=\left(-\mathfrak{n}_{2}, \mathfrak{n}_{1}\right)$ and $(\cdot, \cdot)_{2}$ is the usual scalar product on $\mathbb{R}^{2}$. In this way the configuration $\bar{\sigma}^{\mathfrak{n}}$ is defined on the whole of $\mathbb{Z}^{2 *}$ with all the spins above or on the line $\left\{y:\left(\mathfrak{n}^{\perp}, y\right)_{2}=0\right\}$ being set to +1 and all the remaining spins being set to -1 . Given a configuration $\sigma \in\{-1,1\}_{M}^{\mathbf{S}_{M}^{*}}$ consider a concatenation

$$
\sigma_{M}^{\mathfrak{n}}(y)= \begin{cases}\sigma(y), & \text { if } y \in \mathbf{S}_{M}^{*}, \\ \bar{\sigma}^{\mathfrak{n}}(y), & \text { if } y \in \mathbb{Z}^{2 *} \backslash \mathbf{S}_{M}^{*} .\end{cases}
$$

A bond $b^{*}=(x, y)$ of $\mathbb{Z}^{2 *}$ is called frustrated in $\sigma_{M}^{\mathfrak{n}}$ if $\sigma_{M}^{\mathfrak{n}}(x) \neq \sigma_{M}^{\mathfrak{n}}(y)$. Each dual bond $b^{*}$ intersects (as an interval of $\mathbb{R}^{2}$ ) a unique direct bond $b$ of $\mathbb{Z}^{2}$. Thus, every $\sigma \in\{-1,1\}^{\mathbf{S}_{M}^{*}}$ gives rise to the set

$$
\mathcal{B}_{M}^{\mathfrak{n}}(\sigma)=\left\{b: b^{*} \text { is frustrated }\right\} .
$$

Connected (as subsets of $\mathbb{R}^{2}$ ) components of $\mathcal{B}_{M}^{\mathfrak{n}}(\sigma)$ are called contours. These are microscopic boundaries between regions occupied with spins of different signs. Using a "rounding of corners" procedure [12] contours can be represented as either open or closed self-avoiding curves in $\mathbb{R}^{2}$. Of course, all the contours of $\sigma$ are confined to the strip $\mathbf{S}_{M}=[0, \ldots, M] \times \mathbb{Z}$ except for a unique infinite open contour $\gamma$ which, outside $\mathbf{S}_{M}$ is frozen by the Dobrushin's boundary conditions (1.1) but, of course, varies inside $\mathbf{S}_{M}$ depending on a particular choice of $\sigma$. This portion of $\gamma$ inside $\mathbf{S}_{M}$ which, with a slight abuse of notation, we shall continue to call $\gamma$ is a self-avoiding line connecting the points 0 and $u_{N}$, where (Fig. 1)

$$
\left\lfloor\frac{M}{\mathfrak{n}_{1}} \mathfrak{n}\right\rfloor \triangleq\lfloor N \mathfrak{n}\rfloor \triangleq u_{N}
$$

The contour $\gamma$ models the $\mathfrak{n}$-oriented microscopic interface between co-existing phases of the nearest neighbour Ising model at the inverse temperature $\beta^{*}>\beta_{c}$, where $\beta_{c}=1 / T_{c}$ is the phase transition threshold. The statistics $\mathbb{P}_{N, \beta^{*}}^{\mathfrak{n}}$ of $\gamma$ is read from the Ising Gibbs distribution $\mu_{M, \beta^{*}}^{\mathfrak{n}}$ of $\sigma \in\{-1,+1\}^{\mathbf{S}_{M}^{*}}$ under Dobrushin boundary conditions $\bar{\sigma}^{\mathfrak{n}}$ defined in (1.1). The relation between the strip width $M$ and the projected length of the interface $N$ is given by (1.2) above.

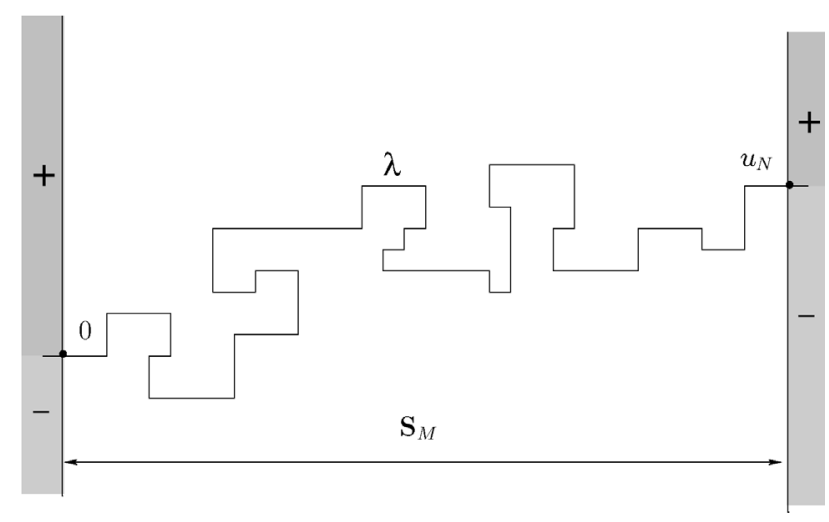

Fig. 1. Phase separation line $\lambda$. 
Our result, which we shall formulate in Subsection 1.6 asserts that under $\mathbb{P}_{N, \beta^{*}}^{\mathfrak{n}}$ an appropriate rescaling of the interface $\gamma$ converges to a Brownian bridge. We prove such convergence for any $\beta^{*}>\beta_{c}$. The fact that the Brownian bridge picture persists all the way to the critical temperature was a well known conjecture.

Over the years statistical properties of phase separation lines have attracted a fair amount of attention. Apart from very specific exact computations of mean magnetization profiles (see e.g. [1,3]) and a study of a scaling limit for a simplified model of random strings [13], the Brownian bridge/random walk structure of interfaces in two dimensions has been previously investigated only for particular models in a perturbative (very low temperature) regime building upon the method of cluster expansions. Important results along these lines include a proof of Gaussian scaling for the interface height in [14] and a full invariance principle for the phase separation line in [15]. The local structure of very low temperature interfaces has been addressed in [5] and in [4].

Very low temperature cluster expansion based approach to a study of phase separation lines culminated in a series of works [12,9-11,16-18]. In these works fluctuations of phase separation lines have been investigated for Ising, Blume-Capel and lattice Widom-Rowlinson interfaces. The so called Dobrushin-Hryniv theory describes fluctuation of interfaces around limiting equilibrium crystal shapes and, furthermore, exactly quantifies the impact of the curvature of the equilibrium shape on the microscopic interface fluctuations in the corresponding directions.

In all the above works the role of very low temperature (very large $\beta$ ) was a purely technical one, for a broad class of models Dobrushin-Hryniv theory should be intrinsic for the whole of the (two) phase co-existing regime. Indeed, in two dimensions interfaces look like essentially one dimensional aggregates of possibly complicated geometric objects and the fact that the original two-dimensional system is away from criticality should, in principle, find an expression in good mixing properties of the induced interface measures. In this respect interfaces in two dimensional low temperature models should resemble connectivity lines/clusters in high temperature models and, subsequently, a fluctuation analysis of the former should fit into a general framework of the Ornstein-Zernike theory.

Our results are based on a recent version of the Ornstein-Zernike theory developed in [6-8]. In the case of the two-dimensional nearest neighbour Ising model, due to very specific self-duality properties, there is a literal correspondence between phase separation lines in a low temperature model and connectivity lines of the high temperature dual model. In particular, an invariance principle for low temperature nearest neighbour Ising phase separation lines is equivalent to a modification of an invariance principle for high temperature connectivity lines in the context of nearest neighbour Ising models confined to lattice strips $\mathbf{S}_{M}$.

In Subsection 1.5 we formulate an invariance principle for connectivity lines in the complete generality of high temperature finite range ferromagnetic Ising type models. A [6]-based proof of an invariance principle for subcritical percolation clusters has been previously given in [20].

Relevant facts from the Ornstein-Zernike theory [7] are collected in Section 2 and are used there for deriving sharp asymptotics for certain partition functions which are needed in order to conclude the proof in Section 3.

\subsection{Random line representation on $\mathbb{Z}^{d}$}

We consider a class of finite range Ising models on $\mathbb{Z}^{d}, d \geqslant 2$ with pair interactions given by the formal Hamiltonian

$$
\sum_{(x, y)} J_{|y-x|} \sigma_{x} \sigma_{y}
$$

where the coupling constants $\left\{J_{x}\right\}$ are non-negative and the set $\left\{x: J_{|x|} \neq 0\right\}$ is bounded. To avoid trivialities we shall also assume that a random walk with jump rates $\left\{J_{x}\right\}$ is irreducible. Let $\beta_{c}$ be the inverse critical temperature corresponding to (1.3). For every $\beta<\beta_{c}$ the following random line representation of two point functions is valid:

$$
\left\langle\sigma_{0} \sigma_{x}\right\rangle_{\beta}=\sum_{\lambda: 0 \rightarrow x} q_{\beta}(\lambda)
$$


where $\langle\cdot\rangle_{\beta}$ it the unique infinite volume Gibbs state for the Hamiltonian (1.3) at $\beta$. We refer to [7] for a discussion of (1.4), in particular for the compatibility conditions employed in the construction of paths $\lambda$.

Given $\mathfrak{n} \in \mathbb{S}^{d-1}$, consider the set $\mathfrak{P}_{N}^{\mathfrak{n}}$ of all compatible paths $\lambda: 0 \rightarrow u_{N} \triangleq\lfloor N \mathfrak{n}\rfloor$. The weights $q_{\beta}(\cdot)$ induce a probability distribution on $\mathfrak{P}_{N}^{\mathfrak{n}}$ :

$$
\mathbb{P}_{N, \beta}^{\mathfrak{n}}(\lambda)=\frac{1}{\left\langle\sigma_{0} \sigma_{u_{N}}\right\rangle_{\beta}} q_{\beta}(\lambda)
$$

We would like to say that under the diffusive scaling, with the direction of $\mathfrak{n}$ playing the role of time and the remaining $(d-1)$ transverse dimensions playing the role of space, the family of measures $\left\{\mathbb{P}_{N, \beta}^{\mathfrak{n}}\right\}$ weakly converges to the distribution of the $(d-1)$-dimensional Brownian bridge.

In order to make such a statement precise we need to recall some facts related to the geometry of the inverse correlation length and to the irreducible decomposition [7,8] of paths from $\mathfrak{P}_{N}^{\mathfrak{n}}$.

\subsection{The geometry of the problem}

The inverse correlation length $\xi_{\beta}$ is defined via

$$
\xi_{\beta}(y)=-\lim _{n \rightarrow \infty} \frac{1}{n} \log \left\langle\sigma_{0} \sigma_{\lfloor n y\rfloor}\right\rangle_{\beta} .
$$

By [2], $\xi_{\beta}$ is an equivalent norm on $\mathbb{R}^{d}$ for every $\beta<\beta_{c}$. It is natural to study fluctuations of random lines $\lambda \in \mathfrak{P}_{N}^{\mathfrak{n}}$ in terms of the geometry of $\xi_{\beta}$. Define

$$
\mathbf{U}_{\beta} \triangleq\left\{x \in \mathbb{R}^{d}: \xi_{\beta}(x) \leqslant 1\right\} \quad \text { and } \quad \mathbf{K}_{\beta} \triangleq \bigcap_{n \in \mathbb{S}^{d-1}}\left\{t \in \mathbb{R}^{d}:(t, n)_{d} \leqslant \xi_{\beta}(n)\right\} .
$$

$\mathbf{U}_{\beta}$ is, of course, just the unit ball in the $\xi_{\beta}$ norm, whereas $\mathbf{K}_{\beta}$ is the effective domain of the convex conjugate (Fenchel transform) of $\xi_{\beta}$. Clearly, $\xi_{\beta}$ could be completely recovered from either of the two compact convex sets above. For example,

$$
\xi_{\beta}(\cdot)=\max _{t \in \partial \mathbf{K}_{\beta}}(t, \cdot)_{d}
$$

where $(\cdot, \cdot)_{d}$ stands for the usual scalar product on $\mathbb{R}^{d}$. As it was proved in [7] $\partial \mathbf{K}_{\beta}$ is locally analytic and has positive Gaussian curvature at any $t \in \partial \mathbf{K}_{\beta}$ for every finite range Ising model at $\beta<\beta_{c}$. In particular, for every $y \in \mathbb{R}^{d} \backslash 0$ the maximum in (1.6) is attained at the unique dual point $t=t_{y}$. For any $t \in \partial \mathbf{K}_{\beta}$ let $\kappa_{i}=\kappa_{i}(t)$, $i=1, \ldots, d-1$, be the principal curvatures of $\partial \mathbf{K}_{\beta}$ at $t$. As we shall see below the magnitude of fluctuations of paths $\lambda \in \mathfrak{P}_{N}^{\mathfrak{n}}$ under $\mathbb{P}_{N, \beta}^{\mathfrak{n}}$ depends on the curvatures $\kappa_{1}, \ldots, \kappa_{d-1}$ at the dual point $t_{\mathfrak{n}}$.

For the rest of this section let us a fix any particular model (interaction potential $\mathbf{J}=\left\{J_{y}\right\}$ ) with the Hamiltonian (1.3) and an inverse temperature $\beta<\beta_{c}(\mathbf{J})$.

\subsection{Irreducible decomposition of paths}

Let $\mathfrak{n} \in \mathbb{S}^{d-1}$ and $t=t_{\mathfrak{n}} \in \partial \mathbf{K}_{\beta}$ is the dual point; $(t, \mathfrak{n})_{d}=\xi_{\beta}(\mathfrak{n})$. Given $\delta>0$, a direction $u \in \mathbb{R}^{d}$ is called forward if

$$
(t, u)_{d}>(1-\delta) \xi_{\beta}(u) .
$$

Let $\mathcal{C}_{\delta}(t)$ be the cone of forward directions. In the sequel we shall fix $\delta$ small enough in order to ensure that any forward direction $u$ has a positive projection on $\mathfrak{n},(\mathfrak{n}, u)_{d}>0$.

Given a compatible path $\lambda=\left(u_{0}, u_{1}, \ldots, u_{n}\right)$ and a number $K$, let us say that $u_{l} \in \lambda$ is a $K$-correct break point if the following two conditions hold: 
(A) $\left(u_{j}, \mathfrak{n}\right)_{d}<\left(u_{l}, \mathfrak{n}\right)_{d}<\left(u_{i}, \mathfrak{n}\right)_{d}$ for all $j<l<i$.

(B) The remaining sub-path $\left(u_{l+1}, \ldots, u_{n}\right)$ lies inside the set

$$
2 K \mathbf{U}_{\beta}\left(u_{l}\right)+\mathcal{C}_{\delta}(t) .
$$

A path is said to be $K$-irreducible if it does not contain $K$-correct break point at all. We use $\mathcal{S}$ to denote the set of all irreducible paths (modulo $\mathbb{Z}^{d}$-shifts). Define also the following three subsets of $\mathcal{S}$ :

$$
\begin{aligned}
& \mathcal{S}_{L}=\left\{\lambda=\left(u_{0}, \ldots, u_{m}\right) \in \mathcal{S}: \forall l>0\left(u_{l}, \mathfrak{n}\right)_{d}<\left(u_{m}, \mathfrak{n}\right)_{d}\right\}, \\
& \mathcal{S}_{R}=\left\{\lambda=\left(u_{0}, \ldots, u_{m}\right) \in \mathcal{S}: \forall l>0\left(u_{l}, \mathfrak{n}\right)_{d}>\left(u_{0}, \mathfrak{n}\right)_{d} \text { and } \lambda \subset K \mathbf{U}_{\beta}\left(u_{0}\right)+\mathcal{C}_{\delta}(t)\right\}, \\
& \mathcal{S}_{0}=\mathcal{S}_{L} \cap \mathcal{S}_{R} .
\end{aligned}
$$

For any path $\gamma=\left(u_{0}, \ldots, u_{m}\right)$ on $\mathbb{Z}^{d}$ set $V(\gamma)=u_{m}-u_{0}$. In other words, $V(\gamma)$ is just the displacement along $\gamma$. We employ the irreducible decomposition of paths $\lambda \in \mathfrak{P}_{N}^{\mathfrak{n}}$,

$$
\lambda=\eta^{L} \amalg \gamma_{1} \amalg \cdots \amalg \gamma_{n} \amalg \eta^{R} .
$$

For any $\lambda \in \mathfrak{P}_{N}^{\mathfrak{n}}$ which has at least two $K$-correct break points the decomposition (1.8) is unambiguously defined by the following set of conditions:

$$
\eta^{L} \in \mathcal{S}_{L}, \quad \eta^{R} \in \mathcal{S}_{R} \quad \text { and } \quad \gamma_{1}, \ldots, \gamma_{n} \in \mathcal{S}_{0} .
$$

In the above definition we have followed [8]. The only difference between (1.8) and the irreducible decomposition employed in [7] is that the break points here are defined with respect to $\mathfrak{n}$-orthogonal hyper-planes instead of $t_{\mathfrak{n}}$-orthogonal hyper-planes. This is to ensure that the displacements along all the $\lambda$-paths which appear in (1.8) have positive projection on the direction of $\mathfrak{n}$.

The renormalization calculus developed in [7] (see Theorem 2.3 there) implies that once the scale $K$ is chosen sufficiently large, paths $\lambda \in \mathfrak{P}_{N}^{\mathfrak{n}}$ with only one $K$-correct break point or without $K$-correct break points at all have exponentially small $\mathbb{P}_{N, \beta}^{\mathfrak{n}}$ probabilities. From now on we shall tacitly assume that such large scale $K$ is fixed and, hence, the notion of irreducible decomposition (1.8) is well defined and the renormalization estimates of [7] apply.

\subsection{Invariance principle for connection paths}

Our approach to the invariance principle is based on the irreducible decomposition (1.8). The following two properties are crucial for the very formulation of the corresponding results:

$$
\min \left\{\left(V\left(\eta^{L}\right), \mathfrak{n}\right)_{d},\left(\left(V\left(\gamma_{1}\right), \mathfrak{n}\right)_{d}, \ldots\left(\left(V\left(\gamma_{n}\right), \mathfrak{n}\right)_{d},\left(V\left(\eta^{R}\right), \mathfrak{n}\right)_{d}\right\}>0\right.\right.
$$

and

$$
\mathbb{P}_{N, \beta}^{\mathfrak{n}}\left(\operatorname{diam}\left(\eta^{L}\right)+\max _{i} \operatorname{diam}\left(\gamma_{i}\right)+\operatorname{diam}\left(\eta^{R}\right)>(\log N)^{2}\right)=\mathrm{O}\left(\frac{1}{N^{\rho}}\right)
$$

for any $\rho>0$.

Property (1.9) simply follows from the definition (1.7) of irreducible paths. Property (1.10) is a straightforward consequence of the abovementioned renormalization result (Theorem 2.3 in [7]) on the mass gap for irreducible connections.

Define $\mathcal{L}_{N}[\lambda]$ to be the linear interpolation in $\mathbb{R}^{d}$ through the vertices

$$
0, V\left(\eta^{L}\right), V\left(\eta^{L}\right)+V\left(\gamma_{1}\right), \ldots, V\left(\eta^{L}\right)+\sum_{1}^{n} V\left(\gamma_{l}\right),\lfloor N \mathfrak{n}\rfloor
$$


By (1.9) the intersection number $\#\left(\mathcal{L}_{N}[\lambda] \cap \mathcal{H}_{h}^{\mathfrak{n}}\right)=1$ for every $h \in\left[0,(\mathfrak{n},\lfloor N \mathfrak{n}\rfloor)_{d}\right]$, where the $(d-1)$-dimensional hyper-plane $\mathcal{H}_{h}^{\mathfrak{n}}$ is defined via

$$
\mathcal{H}_{h}^{\mathfrak{n}}=\left\{u \in \mathbb{R}^{d}:(u, \mathfrak{n})_{d}=h\right\} .
$$

Therefore, there is a natural parameterization of $\mathcal{L}_{N}[\lambda]$ as a function $\Phi_{N}$ on the interval $\left[0,(\mathfrak{n},\lfloor N \mathfrak{n}\rfloor)_{d}\right]$ :

$$
\Phi_{N}(h)=\mathcal{L}_{N}[\lambda] \cap \mathcal{H}_{h}^{\mathfrak{n}}-h \mathfrak{n} .
$$

Finally, we extend the domain of $\Phi_{N}(\cdot)$ to $[0, N]$ by setting it to zero on $\left((\mathfrak{n},\lfloor N \mathfrak{n}\rfloor)_{d}, N\right]$. Notice that $\Phi_{N}(\cdot)$ takes values in the tangent space $\mathbb{R}^{d-1}$ to $\partial \mathbf{K}_{\beta}$ at the dual point $t_{\mathfrak{n}}$. From now on we shall record the values of $\Phi_{N}(\cdot)$ in the orthonormal coordinate system given by the principal curvature directions $\mathfrak{v}_{1}, \ldots, \mathfrak{v}_{d-1}$ of $\partial \mathbf{K}_{\beta}$ at $t=t_{\mathfrak{n}}$.

Since by (1.10) the Hausdorff distance $d_{\mathrm{H}}(\cdot)$ between $\mathcal{L}_{N}[\lambda]$ and $\lambda$ satisfies,

$$
\mathbb{P}_{N, \beta}^{\mathfrak{n}}\left(d_{\mathrm{H}}\left(\mathcal{L}_{N}[\lambda], \lambda\right)>(\log N)^{2}\right)=\mathrm{O}\left(\frac{1}{N^{\rho}}\right)
$$

any limit law for $\Phi_{N}(\cdot)$ which arises on length scales much larger than $(\log N)^{2}$ has a natural interpretation as a limit law for original paths $\lambda$ under the family of probability measures $\left\{\mathbb{P}_{N, \beta}^{\mathfrak{n}}\right\}$.

Define now the rescaled version $\phi_{N}$ of the effective path $\Phi_{N}$ as

$$
\phi_{N}(h)=\frac{1}{\sqrt{N}} \Phi_{N}(N h) ; \quad h \in[0,1]
$$

By construction, $\phi_{N}(h) \in C_{0}^{d-1}[0,1]=\times_{0}^{d-1} C_{0}[0,1]$, where the latter is the space of continuous functions $\phi: 0 \mapsto \mathbb{R}^{d-1}$ satisfying the boundary conditions $\phi(0)=\phi(1)=0$.

Theorem 1.1. The distribution of $\phi_{N}(\cdot)$ under $\mathbb{P}_{N, \beta}^{\mathfrak{n}}$ weakly converges on $C_{0}^{d-1}[0,1]$ to the distribution of

$$
\left(\sqrt{\kappa_{1}} B_{1}(\cdot), \ldots, \sqrt{\kappa_{d-1}} B_{d-1}(\cdot)\right)
$$

where $B_{1}(\cdot), \ldots, B_{d-1}(\cdot)$ are independent standard Brownian bridges on $[0,1]$ and $\kappa_{1}, \ldots, \kappa_{d-1}$ are the principal curvatures of $\partial \mathbf{K}_{\beta}$ at $t_{\mathfrak{n}}$.

\subsection{Phase boundaries in the nearest neighbour 2D Ising model}

In the sequel $\beta^{*}>\beta_{c}$ is an inverse low temperature and $\beta<\beta_{c}$ is the corresponding dual inverse (high) temperature. We refer to [21,23] for a description of the duality relation between high and low temperature two dimensional nearest neighbour Ising models. In particular inverse correlation length $\xi_{\beta}(\cdot)$ at $\beta$ equals to the surface tension $\tau_{\beta^{*}}(\cdot)$ at $\beta^{*}$.

Let us proceed with the notation introduced in the opening Subsection 1.1. Recall that $\mu_{M, \beta^{*}}^{\mathfrak{n}}$ is the Gibbs measure on $\{-1,+1\}_{M}^{\mathbf{S}_{M}^{*}}$ subject to Dobrushin's boundary conditions $\bar{\sigma}^{\mathfrak{n}}$ defined in (1.1). The relation between the strip width $M$ and the projected interface length $N$ was given in (1.2) and we used $\mathbb{P}_{N, \beta *}^{\mathfrak{n}}$ to denote the induced measure on crossing low temperature interfaces $\lambda: 0 \mapsto u_{N}=\lfloor N \mathfrak{n}\rfloor$. However, the set of such interfaces is precisely the set of high temperature connectivity lines inside the direct strip $\mathbf{S}_{M}$. With a slight abuse of notation we shall continue to call this set $\mathfrak{P}_{N}^{\mathfrak{n}}$. Furthermore, by duality,

$$
\mathbb{P}_{N, \beta^{*}}^{\mathfrak{n}}(\lambda)=\frac{1}{\left\langle\sigma_{0} \sigma_{u_{N}}\right\rangle_{N, \beta}} q_{N, \beta}(\lambda) .
$$

Apart from the fact that in the case of phase boundaries all our considerations are confined to two dimensions, the only difference with (1.5) is that now both the weights $q_{N, \beta}$ and the expectation $\langle\cdot\rangle_{N, \beta}$ correspond to the free IsingGibbs state at the inverse temperature $\beta$ on $\mathbf{S}_{M}$. As we shall see, however, the boundary effects have no impact on 
the corresponding invariance principle. In order to decouple these effects from the predominant bulk behavior we shall need, though, a slightly different setup for the decomposition of paths (phase boundaries) $\lambda \in \mathfrak{P}_{N}^{\mathfrak{n}}$. Namely, instead of (1.8) we shall employ:

$$
\lambda=\underline{\eta}^{L} \amalg \underline{\gamma} \amalg \underline{\eta}^{R},
$$

where $\underline{\gamma}=\left(\gamma_{1}, \ldots, \gamma_{n}\right)$ is a string of elements from $\mathcal{S}_{0}$, whereas each of the left and right barriers $\underline{\eta}^{L}=$ $\left(\eta_{1}^{L}, \ldots, \eta_{r}^{L}\right)$ and $\underline{\eta}^{R}=\left(\eta_{1}^{R}, \ldots, \eta_{r}^{R}\right)$ contain exactly

$$
r=\left\lfloor(\log N)^{2}\right\rfloor
$$

elements with $\eta_{1}^{L} \in \mathcal{S}_{L}$ and the rest belonging to $\mathcal{S}_{0}$.

As in the case of (1.10) the renormalization Theorem 2.3 of [7] and the strong triangle inequality of [19,23] imply:

$$
\mathbb{P}_{N, \beta^{*}}^{\mathfrak{n}}\left(\operatorname{diam}\left(\underline{\eta}^{L}\right)+\operatorname{diam}\left(\underline{\eta}^{R}\right)+\max _{i} \operatorname{diam}\left(\gamma_{i}\right)>(\log N)^{4}\right)=\mathrm{O}\left(\frac{1}{N^{\rho}}\right),
$$

for any $\rho>0$.

Similarly to the full-space case define $\mathcal{L}_{N}[\lambda]$ to be the linear interpolation in $\mathbb{R}^{d}$ through the vertices

$$
0, V\left(\underline{\eta}^{L}\right), V\left(\underline{\eta}^{L}\right)+V\left(\gamma_{1}\right), \ldots, V\left(\underline{\eta}^{L}\right)+\sum_{1}^{n} V\left(\gamma_{l}\right),\lfloor N \mathfrak{n}\rfloor,
$$

and then define the rescaled effective path $\phi_{N}(\cdot)$ exactly as in (1.11).

By (1.15) the Hausdorff distance $d_{\mathrm{H}}(\cdot)$ between $\mathcal{L}_{N}[\lambda]$ and $\lambda$ is now bounded as,

$$
\mathbb{P}_{N, \beta}^{\mathfrak{n}}\left(d_{\mathrm{H}}\left(\mathcal{L}_{N}[\lambda], \lambda\right)>(\log N)^{4}\right)=\mathrm{O}\left(\frac{1}{N^{\rho}}\right),
$$

which is still a vanishing quantity under the diffusive scaling.

Theorem 1.2. The distribution of $\phi_{N}(\cdot)$ under $\mathbb{P}_{N, \beta^{*}}^{\mathfrak{n}}$ weakly converges on $C_{0}[0,1]$ to the distribution of

$$
\frac{1}{\sqrt{\tau_{\beta^{*}}^{\prime \prime}(x)+\tau_{\beta^{*}}(x)}} B(\cdot)
$$

where $B(\cdot)$ is the standard Brownian bridge and $\tau_{\beta^{*}}$ is the surface tension of the dual low temperature model (considered here as a function on the unit circle $\left.\mathbb{S}^{1}\right)$. Alternatively, $\left(\tau_{\beta^{*}}^{\prime \prime}(x)+\tau_{\beta^{*}}(x)\right)^{-1}$ is the curvature of the boundary $\partial \mathbf{K}_{\beta}$ of the Wulff shape at the dual point $t_{\mathfrak{n}}, \xi_{\beta}(\mathfrak{n})=\tau_{\beta^{*}}(\mathfrak{n})=\left(t_{\mathfrak{n}}, \mathfrak{n}\right)_{2}$.

\section{Decomposition of path weights}

\subsection{Properties of $q_{\beta}(\cdot)$-weights}

We use notation $q_{\beta, \Lambda}(\cdot)$ for path weights which correspond to the Ising model with Hamiltonian (1.3) at $\beta<\beta_{c}$ with free boundary conditions on $\partial \Lambda$. As before notation $q_{\beta}(\cdot)$ is reserved for infinite volume path weights . A basic reference for the definition and properties of these path weights and for the related path-compatibility conventions is [7,22,23]. As in [7] we shall repeatedly rely on the following decoupling property of the $q_{\beta}(\cdot)$-weights (see Lemma 5.3 in [23] or the proof of Lemma 3.1 in [7]): 
Lemma 2.1. Let $\gamma$ be a (compatible) path and $A, B \subseteq \mathbb{Z}^{2}$. Then,

$$
\frac{q_{\beta, A}(\gamma)}{q_{\beta, B}(\gamma)} \geqslant \exp \left\{-c_{1} \sum_{\substack{x \in \gamma \\ y \in A \Delta B}} \theta^{|y-x|}\right\}
$$

where $c_{1}=c_{1}(\beta)$ and $\theta=\theta(\beta) \in(0,1)$.

Given two compatible paths $\gamma$ and $\lambda$ define the conditional weights

$$
q_{\beta, A}(\gamma \mid \lambda)=\frac{q_{\beta, A}(\gamma \cup \lambda)}{q_{\beta, A}(\lambda)} .
$$

By (2.17)

$$
\frac{q_{\beta, A}(\gamma \mid \lambda)}{q_{\beta, A}(\gamma \mid \eta)} \geqslant \exp \left\{-c_{1} \sum_{\substack{x \in \gamma \\ y \in \lambda \Delta \eta}} \theta^{|y-x|}\right\} \text {. }
$$

\subsection{Strings of paths and semi-norm $\|\cdot\|_{\theta}$}

It would be convenient to consider countable strings $\underline{\lambda}$ of irreducible paths. Any finite string $\underline{\lambda}=\left(\lambda_{1}, \ldots, \lambda_{n}\right)$ is canonically extended to an infinite string by attaching from the right the dummy string of empty paths $\underline{\emptyset}=$ $(\emptyset, \emptyset, \ldots)$;

$$
\left(\lambda_{1}, \ldots, \lambda_{n}\right) \rightarrow\left(\lambda_{1}, \ldots, \lambda_{n}, \emptyset, \emptyset, \ldots\right)=(\underline{\lambda}, \underline{\emptyset}) .
$$

For any two countable strings $\underline{\lambda}, \underline{\alpha}$ define the proximity index

$$
\mathbf{i}(\underline{\lambda}, \underline{\alpha})=\inf \left\{k: \lambda_{k} \neq \alpha_{k}\right\},
$$

and with $\theta=\theta(\beta) \in(0,1)$ from (2.18) define the distance

$$
\mathrm{d}_{\theta}(\underline{\lambda}, \underline{\alpha})=\theta^{\mathbf{i}(\underline{\lambda}, \underline{\alpha})-1} .
$$

Let us say that a function $f$ is locally uniformly Lipschitz continuous if

$$
\|f\|_{\theta} \triangleq \sup _{\mathrm{d}_{\theta}(\underline{\lambda}, \underline{\alpha})<1} \frac{|f(\underline{\lambda})-f(\underline{\alpha})|}{\mathrm{d}_{\theta}(\underline{\lambda}, \underline{\alpha})}<\infty .
$$

\subsection{Uniform Ornstein-Zernike formula}

Let us fix notation: unless it is explicitly mentioned, all paths or strings of paths are assumed to originate at 0 . Given a string $\underline{\lambda}$ and a point $u$, let us use $u \vdash \underline{\lambda}(\underline{\lambda} \dashv u)$ to denote the shift of $\underline{\lambda}$ which starts (respectively ends up) in $u$.

Here is our main input from [7]: Given positive constants $a_{1}, a_{2}$ and $a_{3}$ the OZ formula

$$
\sum_{\underline{\gamma}: V(\underline{\gamma})=\lfloor N \mathfrak{m}\rfloor} q_{\beta}(\underline{\gamma} \mid V(\underline{\gamma}) \vdash \underline{\eta}) f(\underline{\gamma})=\frac{C_{f, \underline{\eta}}(\mathfrak{m})}{\sqrt{N^{d-1}}} \mathrm{e}^{-N \xi_{\beta}(\mathfrak{m})}(1+\mathrm{o}(1)),
$$

holds uniformly in $\mathfrak{m} \in \mathbb{S}^{d-1} \cap \mathcal{C}_{\delta}(t)$, boundary strings $\underline{\eta}$ and functions $f$ satisfying

$$
\frac{1}{a_{1}} \leqslant f(\cdot) \leqslant a_{2} \quad \text { and } \quad\|f\|_{\theta} \leqslant a_{3} .
$$


For every $\underline{\eta}$ and $f$ the function $C_{f, \eta}(\cdot)$ is real analytic on $\mathbb{S}^{d-1} \cap \mathcal{C}_{\delta}(t)$ and, furthermore, there exist $c_{1}=$ $c_{1}\left(a_{1}, a_{2}, \overline{a_{3}}\right)$ and $c_{2}=c_{2}\left(a_{1}, a_{2}, a_{3}\right)$ such that

$$
0<c_{1} \leqslant \inf _{f, \underline{\eta}} C_{f, \underline{\eta}}(\cdot) \leqslant \sup _{f, \underline{\eta}} C_{f, \underline{\eta}}(\cdot) \leqslant c_{2}<\infty,
$$

where both the inf and sup above are over all boundary strings $\underline{\eta}$ and over all functions $f$ satisfying (2.20).

\subsection{Decomposition of $q_{N}\left(\underline{\eta}^{L} \amalg \underline{\gamma} \amalg \underline{\eta}^{R}\right)$}

Let $q_{\vdash, \beta}$ and $q_{\dashv, \beta}$ be the path weights in the right (respectively left) half-spaces $\left\{z \in \mathbb{Z}^{d}: z_{1} \geqslant 0\right\}$ (respectively $\left\{z \in \mathbb{Z}^{d}: z_{1} \leqslant 0\right\}$. Then, in view of the decoupling property (2.18), the super-logarithmic choice of the sizes of barriers $\eta^{L}$ and $\eta^{L}$ in (1.14) ensures:

$$
\frac{q_{N, \beta}\left(\underline{\eta}^{L} \amalg \underline{\gamma} \amalg \underline{\eta}^{R}\right)}{q_{\vdash, \beta}\left(\underline{\eta}^{L}\right) q_{\dashv, \beta}\left(\underline{\eta}^{R}\right)}=q_{\beta}\left(\underline{\gamma} \mid V(\underline{\gamma}) \vdash \underline{\eta}^{R}\right) f_{\underline{\eta}^{L}}(\underline{\gamma})(1+\mathrm{o}(1)),
$$

where

$$
f_{\underline{\eta}^{L}}(\underline{\gamma})=\frac{q_{\beta}\left(\underline{\eta}^{L} \mid V\left(\underline{\eta}^{L}\right) \vdash \underline{\gamma}\right)}{q_{\beta}\left(\underline{\eta}^{L}\right)} .
$$

Notice that $f_{\underline{\eta}} \underline{\underline{L}}^{L}$ satisfies $(2.20)$ for all possible choices of the left barrier $\underline{\eta}^{L}$.

\subsection{Partition function $\mathbf{Z}_{N}(\mathfrak{n})$}

By (1.10) we may restrict attention only to paths $\lambda: 0 \rightarrow u_{N}$ such that each irreducible piece in the decomposition of $\lambda$ has a diameter bounded above by $(\log N)^{2}$. In particular, we may assume that the diameters of the barriers $\underline{\eta}^{R}$ and $\underline{\eta}^{L}$ in $(2.21)$ are bounded above by $(\log N)^{4}$. By the uniform Ornstein-Zernike formula (2.19),

$$
\mathbf{Z}_{N}(\mathfrak{n}) \triangleq \sum_{\lambda \in \mathfrak{P}_{N}^{\mathfrak{n}}} q_{N, \beta}(\lambda)=\frac{C(\mathfrak{n})}{\sqrt{N^{d-1}}} \mathrm{e}^{-N \xi_{\beta}(\mathfrak{n})}(1+\mathrm{o}(1)),
$$

where $C$ is a strictly positive locally analytic function on $\mathbb{S}^{d-1} \cap \mathcal{C}_{\delta}(t)$,

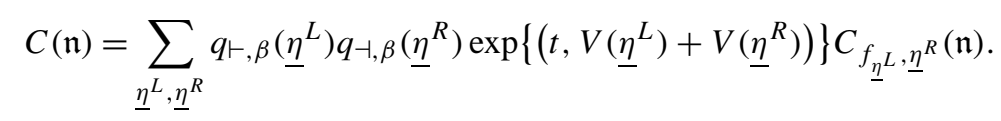

\subsection{Decomposition of $q_{\beta}\left(\underline{\gamma}^{L} \amalg \underline{\alpha} \amalg \underline{\gamma}^{R} \mid V(\underline{\gamma}) \vdash \underline{\eta}^{R}\right)$}

In order to prove convergence of finite dimensional distributions we shall consider a further splitting of $\gamma$ in (1.14) as

$$
\underline{\gamma}=\underline{\gamma}^{L} \amalg \underline{\alpha} \amalg \underline{\gamma}^{R},
$$

where each of the three strings above contain at least $(\log N)^{2}$ irreducible pieces. The induced decomposition of the conditional weights on the right-hand side of (2.21) is:

$$
\frac{q_{\beta}\left(\underline{\gamma}^{L} \amalg \underline{\alpha} \amalg \underline{\gamma}^{R} \mid V(\underline{\gamma}) \vdash \underline{\eta}^{R}\right)}{q_{\beta}(\underline{\alpha})}=q_{\beta}\left(\underline{\gamma}^{L} \mid V\left(\underline{\gamma}_{L}\right) \vdash \underline{\alpha}\right)\left(\underline{\gamma}^{R} \mid V\left(\underline{\gamma}^{R}\right) \vdash \underline{\eta}^{R}\right) f_{\underline{\alpha}}\left(\underline{\gamma}^{R}\right) .
$$


Again, notice that $f_{\underline{\alpha}}$ satisfies (2.20) for all possible choices of the barrier $\underline{\alpha}$. Furthermore,

$$
f_{\underline{\eta}}\left(\underline{\gamma}^{L} \amalg \underline{\alpha} \amalg \underline{\gamma}^{R}\right)=f_{\underline{\eta}^{L}}\left(\underline{\gamma}^{L}\right)(1+\mathrm{o}(1)),
$$

whenever the string $\underline{\gamma}^{L}$ contains at least $(\log N)^{2}$ irreducible pieces (which it will always do).

\subsection{Partition function $\mathbf{Z}_{N}(\mathfrak{n}, v, \underline{\alpha})$}

Define the interior of the strip

$$
\mathbf{S}_{M}^{0}=\left\{y=\left(y_{1}, y_{2}\right) \in \mathbf{S}_{M}: \min \left\{y_{1}, M-y_{1}\right\}>(\log N)^{4}\right\} .
$$

Let us fix $v \in \mathbf{S}_{M}^{0}$ such that both $v$ and $u_{N}-v$ belong to the forward cone $\mathcal{C}_{\delta}(t)$. Fix also an irreducible string $\underline{\alpha}=\left(\alpha_{0}, \alpha_{1}, \ldots, \alpha_{(\log N)^{2}}\right)$. The partition function $\mathbf{Z}_{N}(\mathfrak{n}, v, \underline{\alpha})$ corresponds to the paths $\lambda: 0 \rightarrow u_{N}=\lfloor N \mathfrak{n}\rfloor$ whose irreducible decomposition $\underline{\eta}^{L} \amalg \underline{\gamma} \amalg \underline{\eta}^{R}$ contains $v \vdash \underline{\alpha}$ as a substring. By (2.21), (2.23) and (2.24),

$$
\mathbf{Z}_{N}(\mathfrak{n}, v, \underline{\alpha})=g_{\underline{\alpha}}\left(\mathfrak{n}, \mathfrak{n}_{v}, \mathfrak{n}_{u_{N}-v}\right) \frac{\mathrm{e}^{-\xi_{\beta}(v)-\xi_{\beta}\left(u_{N}-v\right)} q_{\beta}(\underline{\alpha}) \mathrm{e}^{(t, V(\underline{\alpha}))}}{\sqrt{|v|^{d-1}\left|u_{N}-v\right|^{d-1}}}(1+\mathrm{o}(1)),
$$

where, as before $t=t_{\mathfrak{n}}, \mathfrak{n}_{\bullet}=\bullet /|\bullet|$ and given two unit vectors $\mathfrak{m}, \mathfrak{l} \in \mathcal{C}_{\delta}(t) \cap \mathbb{S}^{d-1}$,

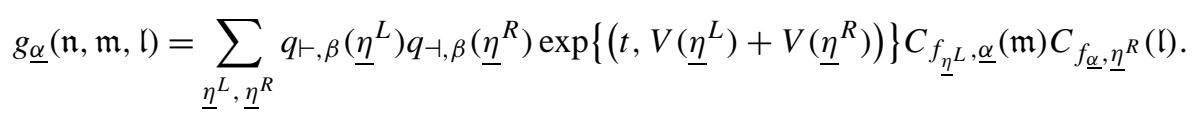

\section{Proof of the invariance principle}

In this section we prove the invariance principle for phase boundaries as stated in Theorem 1.2. Since we rely on the high temperature dual representation (1.13) it would be convenient to write $\mathbb{P}_{N, \beta}^{\mathfrak{n}} \triangleq \mathbb{P}_{N, \beta^{*}}^{\mathfrak{n}}$ for the corresponding interface measures, where, of course, $\beta^{*}>\beta_{c}$ is an inverse low temperature whereas $\beta<\beta_{c}$ is the dual inverse high temperature. The general invariance principle for high temperature connectivity lines (Theorem 1.1) follows along the very same lines and, since we do not have to bother about boundary effects, is actually simpler. The corresponding multi-dimensional curvature computation could be found in [8].

\subsection{The curvature computation}

Let $\mathfrak{n}=\left(\mathfrak{n}_{1}, \mathfrak{n}_{2}\right) \in \mathbb{S}^{1}$ and $\mathfrak{n}^{\perp}=\left(-\mathfrak{n}_{2}, \mathfrak{n}_{1}\right)$ be the unit orthogonal direction. Then, using $\theta(\epsilon)$ to denote the angle of $\mathfrak{n}+\epsilon \mathfrak{n}^{\perp}$;

$$
\theta(\epsilon)=\arctan \frac{\mathfrak{n}_{2}+\epsilon \mathfrak{n}_{1}}{\mathfrak{n}_{1}-\epsilon \mathfrak{n}_{2}}
$$

and recording the derivatives of $\tau_{\beta *}$ in it restriction to the unit circle $\mathbb{S}^{1}$, we obtain:

$$
\begin{aligned}
& \tau_{\beta *}\left(\mathfrak{n}+\epsilon \mathfrak{n}^{\perp}\right)-\tau_{\beta *}(\mathfrak{n})=\sqrt{1+\epsilon^{2}} \tau_{\beta *}(\theta(\epsilon))-\tau_{\beta *}(\theta(0)) \\
& =\frac{1}{2} \epsilon^{2}\left(\tau_{\beta *}(\mathfrak{n})+\tau_{\beta *}^{\prime \prime}(\mathfrak{n})\right)+\tau_{\beta *}^{\prime}(\mathfrak{n})(\theta(\epsilon)-\theta(0))+\mathrm{o}\left(\epsilon^{2}\right) .
\end{aligned}
$$

Let now $u_{N}=\lfloor N \mathfrak{n}\rfloor$ be as in the statement of Theorem 1.2 and, given $h \in(0,1)$ and $a \in \mathbb{R}$, let $v_{N}=N h \mathfrak{n}+$ $\sqrt{N} a \mathfrak{n}^{\perp}$ be an intermediate point inside the interior strip $\mathbf{S}_{M}^{0}$. Then (3.26) (notice that $\theta(\epsilon)+\theta(-\epsilon)-2 \theta(0)=$ $\left.o\left(\epsilon^{2}\right)\right)$ readily implies: 


$$
\begin{aligned}
\tau_{\beta *} & \left(v_{N}\right)+\tau_{\beta *}\left(u_{N}-v_{N}\right)-\tau_{\beta *}\left(u_{N}\right) \\
= & h N\left(\tau_{\beta *}\left(\mathfrak{n}+\frac{a}{h \sqrt{N}} \mathfrak{n}^{\perp}\right)-\tau_{\beta *}(\mathfrak{n})\right)+(1-h) N\left(\tau_{\beta *}\left(\mathfrak{n}-\frac{a}{(1-h) \sqrt{N}} \mathfrak{n}^{\perp}\right)-\tau_{\beta *}(\mathfrak{n})\right) \\
= & \frac{\tau_{\beta *}(\mathfrak{n})+\tau_{\beta *}^{\prime \prime}(\mathfrak{n})}{2 h(1-h)}\left|\frac{v_{N}-N h \mathfrak{n}}{\sqrt{N}}\right|^{2}+\mathrm{O}\left(\frac{1}{\sqrt{N}}\right) .
\end{aligned}
$$

\subsection{Finite dimensional distributions}

We proceed with the setup of Subsection 1.6. Let $\kappa_{\beta}=\left(\tau_{\beta *}(\mathfrak{n})+\tau_{\beta *}^{\prime \prime}(\mathfrak{n})\right)^{-1}$ be the curvature of $\partial \mathbf{K}_{\beta}$ at the dual point $t_{\mathfrak{n}}$. In this section we shall prove convergence of one-dimensional distributions:

Lemma 3.1. For any $h \in(0,1)$ and any $f \in C_{0}(\mathbb{R})$,

$$
\lim _{N \rightarrow \infty} \mathbb{E}_{N, \beta}^{\mathfrak{n}} f\left(\phi_{N}(h)\right)=\mathbb{E} f\left(\sqrt{\kappa_{\beta}} B(h)\right)
$$

As it will be clear from the proof, a generalization to finite dimensional distributions of higher order is straightforward.

It would suffice to show that one can find a sequence $\epsilon_{N} \rightarrow 0$ such that for any $h \in(0,1)$,

$$
\lim _{N \rightarrow \infty} \frac{1}{2 \epsilon_{N}} \int_{h-\epsilon_{N}}^{h+\epsilon_{N}} \mathbb{E}_{N, \beta}^{\mathfrak{n}} f\left(\phi_{N}(s)\right) \mathrm{d} s=\mathbb{E} f\left(\sqrt{\kappa_{\beta}} B(h)\right) .
$$

Given $h \in(0,1)$ and $a \in \mathbb{R}$ define

$$
v_{N}=v_{N}(h, a)=N h \mathfrak{n}+\sqrt{N} a \mathfrak{n}^{\perp} .
$$

Thus,

$$
\left\{\phi_{N}(h)=a\right\}=\left\{v_{N} \in \mathcal{L}_{N}[\lambda]\right\} \triangleq\left\{\lambda \in \mathfrak{P}_{N}^{\mathfrak{n}}\left(v_{N}\right)\right\} .
$$

We record the decomposition (1.14) of each regular (that is satisfying the condition in (1.15)) path $\lambda \in \mathfrak{P}_{N}^{\mathfrak{n}}\left(v_{N}\right)$ as, see Fig. 2,

$$
\lambda=\underline{\eta}^{L} \amalg \underline{\gamma}^{L} \amalg \underline{\alpha} \amalg \underline{\gamma}^{R} \amalg \underline{\eta}^{R},
$$

where the barrier $\underline{\alpha}=\left(\alpha_{0}, \alpha_{1}, \ldots, \alpha_{r}\right)$ (recall $\left.r=\left\lfloor(\log N)^{2}\right\rfloor\right)$ is unambiguously defined by the condition

$$
v_{N} \in\left(V\left(\underline{\eta}^{L}\right)+V\left(\underline{\gamma}^{L}\right), V\left(\underline{\eta}^{L}\right)+V\left(\underline{\gamma}^{L}\right)+V\left(\alpha_{0}\right)\right),
$$

where $[u, v)$ is the semi-open linear segment in $\mathbb{R}^{2}$ with end-points at $u$ and $v$. In the notation of Subsections 2.5 and 2.7,

$$
\mathbb{P}_{N, \beta}^{\mathfrak{n}}\left(v_{N} \in \mathcal{L}_{N}[\lambda]\right)=\sum_{\substack{(v, \underline{\alpha}): \\ v_{N} \in\left[v, v+V\left(\alpha_{0}\right)\right)}} \frac{\mathbf{Z}_{N}(\mathfrak{n}, v, \underline{\alpha})}{\mathbf{Z}_{N}(\mathfrak{n})}
$$

By (1.15) we can restrict attention to $v$-s satisfying $\left|v-v_{N}\right| \leqslant(\log N)^{4}$. Then the asymptotic expressions (2.22) and (2.25) and the curvature computation (3.27) yield:

$$
\mathbb{P}_{N, \beta}^{\mathfrak{n}}\left(v_{N} \in \mathcal{L}_{N}[\lambda]\right)(1+\mathrm{o}(1))=\frac{1}{\sqrt{N h(1-h)}} \exp \left\{-\frac{\left|v_{N}-N h \mathfrak{n}\right|^{2}}{2 N h(1-h) \kappa_{\beta}}\right\} \sum_{\substack{(v, \underline{\alpha}): \\ v_{N} \in\left[v, v+V\left(\alpha_{0}\right)\right)}} G(\underline{\alpha}),
$$




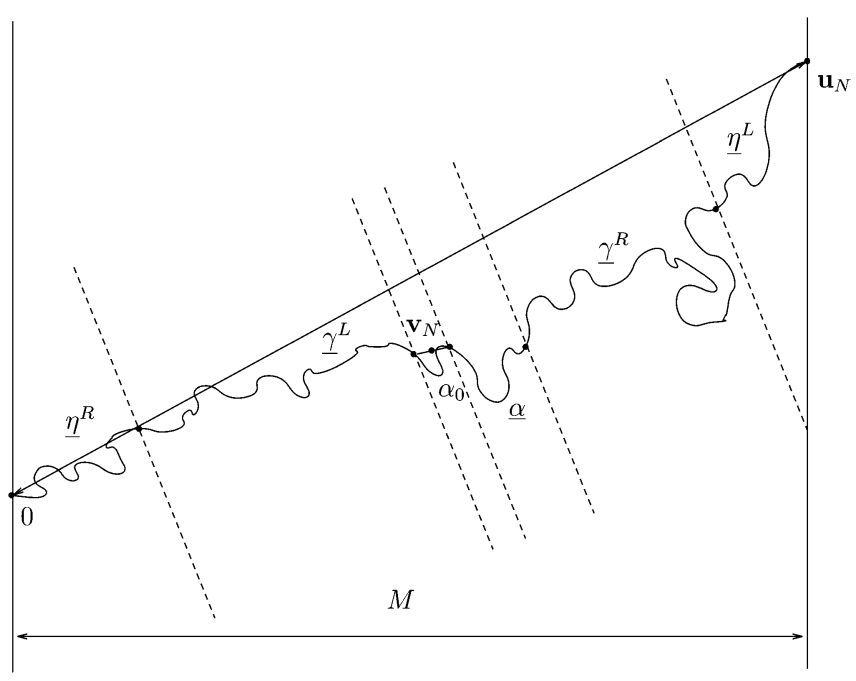

Fig. 2. Decomposition of a path $\lambda \in \mathfrak{P}_{N}^{\mathfrak{n}}\left(v_{N}\right)$. The point $v_{N}$ belongs to the segment $\left[V\left(\underline{\eta}^{L}\right)+V\left(\underline{\gamma}^{L}\right), V\left(\underline{\eta}^{L}\right)+V\left(\underline{\gamma}^{L}\right)+V\left(\alpha_{0}\right)\right)$. $\underline{\alpha}=\left(\alpha_{0}, \ldots, \alpha_{r}\right)$ is a decoupling barrier.

where $G(\underline{\alpha})=g_{\underline{\alpha}}(\mathfrak{n}, \mathfrak{n}, \mathfrak{n}) q_{\beta}(\underline{\alpha}) \mathrm{e}^{(t, V(\underline{\alpha}))}$ in the notation of $(2.25)$.

Consequently,

$$
\mathbb{E}_{N, \beta}^{\mathfrak{n}} f\left(\phi_{N}(h)\right)(1+\mathrm{o}(1))=\sum_{\substack{(v, \underline{\alpha}): \\\left[v, v+V\left(\alpha_{0}\right)\right) \cap \mathcal{H}_{N h}^{\mathfrak{n}} \neq \emptyset}} f\left(\frac{v-(v, \mathfrak{n})_{2} \mathfrak{n}}{\sqrt{N}}\right) \frac{\mathrm{e}^{-\left|v-(v, \mathfrak{n})_{2} \mathfrak{n}\right|^{2} /\left(2 N h(1-h) \kappa_{\beta}\right)}}{\sqrt{N h(1-h)}} G(\underline{\alpha}),
$$

where we have again relied on the possibility to restrict attention to $\left|V\left(\alpha_{0}\right)\right| \leqslant(\log N)^{2}$.

We are now in a position to derive (3.29): Choose $\epsilon_{N}$ in such a way that $N \epsilon_{N} \gg(\log N)^{2}$. Then,

$$
\begin{aligned}
(1 & +\mathrm{o}(1)) \int_{h-\epsilon_{N}}^{h+\epsilon_{N}} \mathbb{E}_{N, \beta}^{\mathfrak{n}} f\left(\phi_{N}(s)\right) \mathrm{d} s \\
& =\frac{1}{\sqrt{N h(1-h)}} \sum_{v, \underline{\alpha}} f\left(\frac{v-(v, \mathfrak{n})_{2} \mathfrak{n}}{\sqrt{N}}\right) \mathrm{e}^{-\left|v-(v, \mathfrak{n})_{2} \mathfrak{n}\right|^{2} /\left(2 N h(1-h) \kappa_{\beta}\right)} G(\underline{\alpha}) \int_{h-\epsilon_{N}}^{h+\epsilon_{N}} \mathbb{1}_{\left\{(v, \mathfrak{n})_{2} \leqslant s N \leqslant\left(v+V\left(\alpha_{0}\right), \mathfrak{n}\right)_{2}\right\}} \mathrm{d} s \\
& =\frac{C(\mathfrak{n})}{\sqrt{h(1-h)}} \frac{1}{N^{3 / 2}} \sum_{N\left(h-\epsilon_{N}\right) \leqslant(v, \mathfrak{n})_{2} \leqslant N\left(h+\epsilon_{N}\right)} f\left(\frac{v-(v, \mathfrak{n})_{2} \mathfrak{n}}{\sqrt{N}}\right) \mathrm{e}^{-\left|v-(v, \mathfrak{n})_{2} \mathfrak{n}\right|^{2} /\left(2 N h(1-h) \kappa_{\beta}\right)}+\mathrm{o}\left(\epsilon_{N}\right),
\end{aligned}
$$

where

$$
C(\mathfrak{n})=\sum_{\underline{\alpha}}\left(V\left(\alpha_{0}\right), \mathfrak{n}\right)_{2} G(\underline{\alpha}) .
$$

In fact, $C(\mathfrak{n})=1 / \sqrt{2 \pi}+\mathrm{o}(1)$. Indeed, the expression above is just the Riemannian sum for the integral

$$
2 \epsilon_{N} \int_{-\infty}^{\infty} f(z) \frac{\mathrm{e}^{-z^{2} /\left(2 h(1-h) \kappa_{\beta}\right)}}{\sqrt{2 h(1-h) \kappa_{\beta}}} \mathrm{d} z+\mathrm{o}\left(\epsilon_{N}\right)
$$

and (3.29) follows. 


\subsection{Tightness}

In this section we prove tightness of the $\varphi_{N}$. In order to simplify the proof we introduce a modification of $\varphi_{N}$, which is a linear approximation of $\varphi_{N}$ on a larger mesoscopic scale with step size $S_{N}=\left\lfloor N^{1 / 2-\delta}\right\rfloor$.

The number of irreducible components in the decomposition (1.8) of a path $\lambda$ before the $i$-th step on the mesoscopic grid will be denoted by $T_{N, i}(\lambda)$

$$
T_{N, i}(\lambda)=\max \left\{k:\left(V\left(\eta^{L}\right), \mathfrak{n}\right)_{2}+\sum_{j=0}^{k}\left(V\left(\lambda_{j}\right), \mathfrak{n}\right)_{2} \leqslant i S_{N}\right\} ;
$$

the coordinates of the displacement of the first $T_{N, i}(\lambda)$ irreducible components in the path $\lambda$ denoted by

$$
C_{N, i}(\lambda)=V\left(\eta^{L}\right)+\sum_{j=0}^{T_{N, i}} V\left(\lambda_{j}\right)
$$

Our mesoscopic linear approximation of $\lambda: 0 \mapsto u_{N}$, which will be denoted by $\overline{\mathcal{L}}_{N}$, is the linear interpolation through the points

$$
0, \bar{V}_{1}, \ldots, \bar{V}_{\left\lfloor N / S_{N}\right\rfloor-1}, \quad u_{N} \in \mathbb{R}^{2},
$$

where the $\bar{V}_{i}$ is recorded in $\left(\mathfrak{n}, \mathfrak{n}^{\perp}\right)$-coordinate system as:

$$
\bar{V}_{i}=i S_{N} \mathfrak{n}+\left(C_{N, i-1}(\lambda), \mathfrak{n}^{\perp}\right)_{2} \mathfrak{n}^{\perp} .
$$

The above definition of the vertices $\left\{\bar{V}_{i}\right\}$ is tuned in such a way that their $\mathfrak{n}$ (time) projections live on the step- $S_{N}$ mesoscopic grid.

Similarly to the rescaled version of the effective path $\varphi_{N}$, we define a rescaled version of the approximation by $\bar{\varphi}_{N}$. Note that the irreducible decomposition procedure along with the restriction on the length of the irreducible components by $(\log N)^{4}$ imply that

$$
\lim _{N \rightarrow \infty} \mathrm{d}_{\mathrm{H}}\left(\varphi_{N}, \bar{\varphi}_{N}\right)=0,
$$

where $\mathrm{d}_{\mathrm{H}}(\cdot, \cdot)$ is Hausdorff distance.

To prove tightness of $\bar{\varphi}_{N}$ it is enough to show that

$$
\mathbb{E}_{N, \beta}^{\mathfrak{n}}\left|\bar{\varphi}_{N}\left(h_{2}\right)-\bar{\varphi}_{N}\left(h_{1}\right)\right|^{4} \leqslant C\left|h_{2}-h_{1}\right|^{2}
$$

uniformly on $0 \leqslant h_{1}<h_{2} \leqslant 1$.

Lemma 3.2. There exist $c_{1}, c_{2}>0$ such that

$$
\mathbb{P}_{N, \beta}^{\mathfrak{n}}\left(\left|\bar{\varphi}_{N}(h)\right| \geqslant a \sqrt{h}\right)<c_{1} \exp \left(-c_{2} \min \left(a^{2}, a\right)\right)
$$

holds uniformly on $h \in(0,1)$ and $a>0$.

Proof. By definition of $\bar{\varphi}_{N}$, we have

$$
\mathbb{P}_{N, \beta}^{\mathfrak{n}}\left(\left|\bar{\varphi}_{N}(h)\right| \geqslant a \sqrt{h}\right)=\sum_{\substack{(v, \underline{\alpha}): \\\left|\left(v, \mathfrak{n}^{\perp}\right)_{2}\right| \geqslant a \sqrt{h N} \\(v, \mathfrak{n})_{2} \leqslant h N<\left(v+V\left(\alpha_{0}\right), \mathfrak{n}\right)_{2}}} \frac{\mathbf{Z}_{N}(\mathfrak{n}, v, \underline{\alpha})}{\mathbf{Z}_{N}(\mathfrak{n})} ;
$$

moreover it is sufficient to prove (3.35) for $h \in(0,1) \cap\left(\mathbb{Z} \cdot \frac{S_{N}}{N}\right)$. 
We divide the sum in (3.36) into two regimes: the very large deviations regime when either $v \notin \mathcal{C}_{\delta}(t)$ or $u_{N}-v \notin$ $\mathcal{C}_{\delta}(t)$ and the large to moderate deviations regime when both $v$ and $u_{N}-v$ belong to the forward cone $\mathcal{C}_{\delta}(t)$. It enough to show that the bound (3.35) holds when we restrict the sum to each of the regimes.

Let $v \notin \mathcal{C}_{\delta}(t)$. Then the renormalization Theorem 2.3 of [7] readily implies: There exists $c_{3}>0$ such that,

$$
\sum_{\underline{\eta}^{L} \amalg \underline{\gamma}: 0 \mapsto u} q_{\beta, N}\left(\underline{\eta}^{L} \amalg \underline{\gamma} \mid \underline{\alpha}\right) \leqslant \mathrm{e}^{-\xi_{\beta}(u)-2 c_{3}|u|},
$$

uniformly in $\underline{\alpha}$ and in $|u-v| \leqslant(\log N)^{2}$. Consequently,

$$
\frac{\mathbf{Z}_{N}(\mathfrak{n}, v, \underline{\alpha})}{\mathbf{Z}_{N}(\mathfrak{n})} \leqslant \exp \left\{-\left(\xi_{\beta}(v)+\xi_{\beta}\left(u_{N}-v\right)-\xi_{\beta}\left(u_{N}\right)\right)-c_{3}|v|\right\},
$$

and, in view of convexity of $\xi_{\beta}$, a substitution of the above estimate to (3.36) clearly implies (3.35). A completely similar argument applies in the case of $u_{N}-v \notin \mathcal{C}_{\delta}(t)$.

It remains to consider the case when both $v$ and $u_{N}-v$ belong to the forward cone $\mathcal{C}_{\delta}(t)$. In this case we are entitled to use the asymptotic formula (2.25). In fact, since the strict triangle inequality of $[19,23]$ implies that

$$
\xi_{\beta}(v)+\xi_{\beta}\left(u_{N}-v\right)-\xi_{\beta}\left(u_{N}\right) \geqslant c_{4} \frac{\left|(v, \mathfrak{n})_{2}\right|^{2}}{N},
$$

we can restrict attention only to the case of moderate deviations $\left|(v, \mathfrak{n})_{2}\right| \leqslant N^{1 / 2+\delta}$. For such values of $v$ we, exactly as in (3.31), infer that the right-hand side of (3.36) is bounded above by

$$
\frac{C(\mathfrak{n})}{\sqrt{N h(1-h)}} \sum_{\sqrt{N b} \in(\sqrt{N h} a, \infty) \cap \mathbb{Z}} \exp \left\{-\frac{b^{2}}{2 h(1-h) \kappa_{\beta}}\right\}(1+\mathrm{o}(1)),
$$

where $C(\mathfrak{n})$ is given in (3.32), and (3.35) follows from the usual Gaussian summation procedure.

The decomposition bounds imply that

$$
q_{N, \beta}\left(\underline{\eta}^{L} \amalg \underline{\gamma}_{1} \amalg \underline{\gamma}_{2} \amalg \underline{\eta}^{R}\right) \leqslant c_{4} q_{N, \beta}\left(\underline{\eta}^{L} \amalg \underline{\gamma}_{2} \amalg \underline{\gamma}_{1} \amalg \underline{\eta}^{R}\right)
$$

which along with Lemma 3.2 yields: there exist $C_{2}, C_{3}>0$ such that

$$
\mathbb{P}_{N, \beta}^{\mathfrak{n}}\left(\frac{\left|\bar{\varphi}_{N}\left(h_{2}\right)-\bar{\varphi}_{N}\left(h_{1}\right)\right|}{\left|h_{2}-h_{1}\right|} \geqslant a\right) \leqslant C_{3} \exp \left(-C_{2} \min \left(a^{2}, a\right)\right)
$$

holds uniformly on $0 \leqslant h_{1}<h_{2} \leqslant 1$ and $a>0$.

Finally, the bound (3.37) gives us the tightness criterion (3.34) for $\bar{\varphi}_{N}$, which along with (3.33) implies the tightness of the $\varphi_{N}$.

\section{References}

[1] D.B. Abraham, P. Reed, Interface profile of the Ising ferromagnet in two dimensions, Commun. Math. Phys. 49 (1) (1976) $35-46$.

[2] M. Aizenman, D.J. Barsky, R. Fernández, The phase transition in a general class of Ising-type models is sharp, J. Statist. Phys. 47 (3/4) (1987) 342-374.

[3] A. Akutsu, N. Akutsu, Relationship between the anisotropic interface tension, the scaled interface width and the equilibrium shape in two dimensions, J. Phys. A: Math. Gen. 19 (1986) 2813-2820.

[4] J. Bricmont, J. Fröhlich, Statistical mechanical methods in particle structure analysis of lattice field theories. II. Scalar and surface models, Commun. Math. Phys. 98 (4) (1985) 553-578.

[5] J. Bricmont, J.L. Lebowitz, C.-E. Pfister, On the local structure of the phase separation line in the two-dimensional Ising system, J. Statist. Phys. 26 (2) (1981) 313-332.

[6] M. Campanino, D. Ioffe, Ornstein-Zernike theory for the Bernoulli bond percolation on $\mathbb{Z}^{d}$, Ann. Probab. 30 (2002) $652-682$. 
[7] M. Campanino, D. Ioffe, Y. Velenik, Ornstein-Zernike theory for finite range Ising models above $T_{c}$, Probab. Theory Related Fields 125 (3) (2003) 305-349.

[8] M. Campanino, D. Ioffe, Y. Velenik, Random path representation and sharp correlations asymptotics at high-temperatures, in: Stochastic Analysis on Large Scale Interacting Systems, in: Adv. Stud. Pure Math., vol. 39, Math. Soc. Japan, Tokyo, 2004, pp. $29-52$.

[9] R. Dobrushin, A statistical behaviour of shapes of boundaries of phases, in: R. Kotecký (Ed.), Phase Transitions: Mathematics, Physics, Biology ..., World Scientific, Singapore, 1993, pp. 60-70.

[10] R. Dobrushin, O. Hryniv, Fluctuations of shapes of large areas under paths of random walks, Probab. Theory Related Fields 105 (4) (1996) 423-458.

[11] R. Dobrushin, O. Hryniv, Fluctuations of the phase boundary in the 2D Ising ferromagnet, Commun. Math. Phys. 189 (2) (1997) $395-445$.

[12] R. Dobrushin, R. Kotecký, S. Shlosman, Wulff construction. A global shape from local interaction, Transl. Math. Monographs, vol. 104, American Mathematical Society, Providence, RI, 1992.

[13] R. Durrett, On the shape of a random string, Ann. Probab. 7 (1978) 1014-1027.

[14] G. Gallavotti, The phase separation line in the two-dimensional Ising model, Commun. Math. Phys. 27 (1972) $103-136$.

[15] Y. Higuchi, On some limit theorem related to the phase separation line in the two-dimensional Ising model, Z. Wahrsch. Verw. Gebiete 50 (3) (1979) 287-315.

[16] Y. Higuchi, J. Murai, J. Wang, The Dobrushin-Hryniv theory for the two-dimensional lattice Widom-Rowlinson model, Adv. Stud. Pure Math., in press.

[17] O. Hryniv, On local behaviour of the phase separation line in the 2D Ising model, Probab. Theory Related Fields 110 (1) (1998) 91-107.

[18] O. Hryniv, R. Kotecký, Surface tension and the Ornstein-Zernike behaviour for the 2D Blume-Capel model, J. Statist. Phys. (2001).

[19] D. Ioffe, Large deviations for the 2D Ising model: a lower bound without cluster expansions, J. Statist. Phys. 74 (1-2) (1994) 411-432.

[20] Y. Kovchegov, The Brownian bridge asymptotics in the subcritical phase of Bernoulli bond percolation model, Markov Process. Related Fields 10 (2) (2004) 327-344.

[21] Ch.-E. Pfister, Large deviations and phase separation in the two-dimensional Ising model, Helv. Phys. Acta 64 (7) (1991) $953-1054$.

[22] C.-E. Pfister, Y. Velenik, Large deviations and continuum limit in the 2D Ising model, Probab. Theory Related Fields 109 (1997) $435-506$.

[23] C.-E. Pfister, Y. Velenik, Interface, surface tension and reentrant pinning transition in the 2D Ising model, Commun. Math. Phys. 204 (2) (1999) 269-312. 